\title{
A Mathematical Model of Lubricant Film Flow Velocity on a Belt Type Oil Skimmer in a Part of Wastewater Treatment Process Using a Finite Difference Method with Quasi-Newton Iterative Technique
}

\author{
Pantira Klankaew ${ }^{1,2}$, Kaboon Thongtha ${ }^{3}$, Siripawn H Winter ${ }^{1,2}$, Pornchai Chaisanit $^{1,2}$, \\ Kanchana Kumnungkit ${ }^{1,2}$, Nopparat Pochai ${ }^{1,2, *}$ \\ ${ }^{1}$ Department of Mathematics, King Mongkut's Institute of Technology Ladkrabang, Thailand \\ ${ }^{2}$ Centre of Excellence in Mathematics CHE, Si Ayutthaya Rd, Bangkok 10400, Thailand \\ ${ }^{3}$ Faculty of Information Science and Technology, Mahanakorn University of Technology, Thailand
}

Received February 7, 2020; Revised April 15, 2020; Accepted April 27, 2020

Copyright $(2020$ by authors, all rights reserved. Authors agree that this article remains permanently open access under the terms of the Creative Commons Attribution License 4.0 International License

\begin{abstract}
Oil skimmer is a useful tool in recovering all types of floating waste oils, greases and fats from water surfaces. Lubricant film flow velocity approximation is an important problem of oil skimmer belt speed adjustment. The adjustment belt speed level is up to several physical parameters of oil types. A thin lubricant film flow velocity on a moving belt oil skimmer can be modeled in a form of a nonlinear differential equation as a boundary value problem. The model is providing the lubricant film flow velocity in each thickness layers. In this research, a centered in space finite difference method and a Quasi-Newton iterative method are proposed to approximate the solutions of the nonlinear thin lubricant film flow velocity model. Their numerical simulations of a thin lubricant film flow velocity on a moving oil skimmer belt with varied physical parameters are investigated. The proposed numerical techniques give good agreement approximated solutions in several moving belts speed levels with the external force factor. These are then useful to achieve the optimum belt oil skimmer speed for each lubricant type.
\end{abstract}

Keywords Lubricant Film, Velocity, Oil Skimmer, Finite Difference Method, Quasi-Newton Method

\section{Introduction}

An oil skimmer is a mechanical gadget that isolates oil or particles drifting on a fluid surface and are most normally utilized for oil slick remediation, as a piece of sleek water treatment frameworks, expelling oil from machine apparatus coolant and expelling oil from watery parts washers. Oil skimming devices come in a variety of types with varying features. Oil Skimmers can be used for tanks, pits, ponds, lagoons, basins, wash systems, API separators, clarifiers, sumps and many more purposes. The Non-Newtonian fluid is referring a fluid whose viscosity is variable based on the applied stress or force. Behavior of Newtonian fluids like water can be described exclusively by temperature and pressure. The research of Non-Newtonian fluid is bringing up some interesting result when combined theory with mechanical engineering. Most of the scientific problems in fluid mechanics are modeled by nonlinear differential equations. It is well known that exact solutions of these nonlinear boundary value problems are difficult to obtain. Therefore, numerical solutions methods and analytical solutions methods are used to handle such type of problems.

Oil skimmers are pieces of equipment which remove oil that floats on a fluid surface. Oil skimmers generally work because they are made of materials to which oil is more likely to stick than the fluid on which it floats. The fluid has very little appeal for oil skimmers, at the same time. Oil skimmers are usually anything needed to remove oil from a liquid. Nevertheless, in some situations oil skimmers may be used for pretreatment of a fluid. In this situation, the oil skimmers strip as much of the oil as possible before taking more expensive and time-consuming steps. Pre-treating oil skimmers with the solvent reduces the overall expense of washing the material. 
There are two basic oil skimmers types. One type is used for filtering and removing oil, debris and undesirable materials. The other type is used to reclaim or capture oil in a usable condition. The second type of skimmer is usually used to clean up oil spills at Water's plants, refineries, and bodies. Oil skimmers uses include in-plant pumps, tanks, and refrigerant systems, as well as outdoor cooling and settling ponds. Critical requirements to consider when selecting oil skimmers include power, the rate of removal or recovery and total flow. When measuring power in gallons, flow is measured in gallons per minute (gpm), and the rate of removal or recovery is expressed in gallons per hour (gph). There are several different types of oil skimmers, though all designs are based on gravity laws and surface tension for working. Belt oil skimmers make use of a stainless steel belt. This belt is lowered to the liquid to scrub. Then the belt passes through special wiper blades, removing the oil from both sides of the liquid as it passes.

The shear stress is dependent on the share rate in most fluid food products; therefore, nonlinear flow curve results and a unique viscosity are no longer adequate for characterizing the fluid. Many fluids, such as molten plastics, polymers, and slurries in their flow behavior are non-Newtonian. The basic governing equations for such fluid movement are highly nonlinear differential equations that have no general solution, and only a limited number of exact solutions for specific problems have been developed. Researchers and scientists have developed various techniques to solve practical problems in engineering and mathematics; researchers and scientists have developed numerous numerical techniques, that is, finite difference method (FDM), finite volume approach, control-volume-based finite element method (CVFEM), lattice Boltzmann method (LBM), and analytical techniques, that is, variational iteration method (VIM), perturbation method (PM), homotopy perturbation method (HPM), HPM-Pade technique, homotopy analysis method (HAM), optimal homotopy analysis method (OHAM), optimal homotopy perturbation method (OHPM), and some other techniques, to overcome nonlinearity and get numerical and analytical solutions.

In applying this approach to a broad class of linear, nonlinear, and partial differential equations and integral equations a considerable amount of research work has been expended. Several important problems in applied science and engineering have been successfully solved by using ADM to their greater precision. A useful benefit of the $\mathrm{ADM}$ is that it has proven to be a reliable alternative to the approach used in the Taylor series and other series. This approach was used to obtain analytical and approximate solutions for a broad class of linear and nonlinear, differential and integral equations, homogeneous or inhomogeneous, with constant coefficients, or with variable coefficients. The Adomian decomposition method is comparatively simpler to program in engineering problems than other series approaches, and offers definitions for immediate and observable solutions without linearization, disruption or control of the problem, while the solution's physical behavior remains unchanged.

In [1] and [2], they have proposed the solution of the thin film flow of a third grade fluid on a moving belt model by using the homotopy perturbation method. In [3] and [4], they have developed a generalized approximation method (GAM) to obtain a solution of a thin film flow of a third grade fluid on a moving belt. The GAM generates a monotone sequence of solutions of linear problems. The sequence of solutions of linear problems converges monotonically and rapidly to a solution of the original nonlinear problem. We present some numerical simulations to illustrate and confirm our results. In [5], they have investigated a thin film flow of a third grade fluid on a moving belt using a powerful and relatively new approximate analytical technique known as optimal homotopy asymptotic method (OHAM). The variation of velocity profile for different parameters is compared with the numerical values obtained by Runge-Kutta Fehlberg fourth-fifth order method and with Adomian Decomposition Method (ADM). An interesting result of the analysis is that the three terms OHAM solution is more accurate than five terms of the ADM solution and this thus confirms the feasibility of the proposed method.

In [7], the main objective is to analyze the effect of volume fraction of nanoparticles on MHD stagnation point flow towards a moving surface with convective heat and mass transfer parameters. The governing boundary layer equations have been transformed to a two-point boundary value problem using similarity variables. These similarity equations were solved numerically using Runge-Kutta Fehlberg fourth-fifth order method. The effects of governing parameters on the dimensionless velocity, temperature, and particle concentration as well as on local skin friction and Nusselt and Sherwood numbers have been investigated. In [8], a numerical treatment for axisymmetric flow and heat transfer due to a stretching cylinder under the influence of a uniform magnetic field and prescribed surface heat flux is presented. Numerical results are obtained for dimensionless velocity, temperature, skin friction coefficient and Nusselt number for several values of the suction/injection, magnetic and curvature parameters as well as the Prandtl number. The present study reveals that the controlling parameters have strong effects on the physical quantities of interest. It is seen that the magnetic field enhances the dimensionless temperature inside the thermal boundary layer, whereas it reduces the dimensionless velocity inside the hydrodynamic boundary layer. Heat transfer rate reduces, while the skin friction coefficient increases with magnetic field.

Recently there has been a growing interest in the study of the differential form fluid flow and heat transfer. These fluid flows and heat transfer have large applications in heat exchangers, the method of screw extrusion, electronics 
cooling and many others. Extensive studies describing the flows of second grade fluids have been under taken in various flow geometries and under several assumptions. In [9], analytical solutions were identified for the unstable flow of a third-grade fluid over a moved plate. The relevant problem is formulated using the law of a third-grade fluid of modified Darcy. They present and discuss two types of analytical solutions. It also presents a numerical solution. Non-Newtonian fluids have gained significant interest because in industry and technology they are found. The flows of such fluids in computational fluid dynamics and heat transfer provide the benchmark issues. The resulting structures describe the ideal problems for testing various numerical methods as well as the validity of constitutive equations used to classify non-Newtonian fluid's rheological properties. As a result, a wealth of literature exists on such flow covering a wide range of fluids and governing parameters.

In [10], the steady flow of an incompressible, third-grade fluid in helical screw rheometer (HSR) is studied by "unwrapping or flattening" the channel, lands, and the outside rotating barrel. The geometry is approximated as a shallow infinite channel, by assuming that the width of the channel is large as compared to the depth. Using a transformation, the developed nonlinear second-order coupled differential equations are reduced to single differential equation. Analytical expressions for the velocity profiles and volume flow rates are calculated using Adomian decomposition method. The objective of this paper is to study the flow of third-grade fluid in helical screw rheometer (HSR) where the effects of curvature and also of flights are neglected by assuming that the helical channel is "unwrapped." The geometry is approximated as a shallow infinite channel

In this research, a couple of two numerical techniques such as a centered in space finite difference technique and the Quasi-Newton iterative method is used to approximate the solutions of a nonlinear mathematical model of lubricant film flow velocity on a belt type oil skimmer. The proposed numerical techniques give good agreement approximated solutions in several moving belts speed levels.

\section{The Lubricant Film Flow Velocity on a Belt Type Oil Skimmer Model}

The lubricant film flow velocity on a belt type oil skimmer is governed by the following nonlinear boundary value problem. $[1,2,4,5]$.

$$
\frac{d^{2} v}{d x^{2}}+6 \frac{\left(\beta_{2}+\beta_{3}\right)}{\mu}\left(\frac{d v}{d x}\right)^{2} \frac{d^{2} v}{d x^{2}}-\frac{\rho g}{\mu}=0,
$$

for all $0 \leq x \leq \delta$, subject to the boundary conditions

$$
v(0)=u_{l}
$$

$$
v^{\prime}(\delta)=u_{r}
$$

where $v$ is the fluid velocity $(\mathrm{m} / \mathrm{s}), \rho$ is the density $\left(\mathrm{Kg} / \mathrm{m}^{3}\right), \quad \mu$ is the dynamic viscosity $(\mathrm{Pa} \cdot \mathrm{S}), \beta_{2}, \beta_{3}$ are the material constants of the third grade fluid (non-units), $g$ is the gravity acceleration $\left(\mathrm{m} / \mathrm{s}^{2}\right), \delta$ is the uniform thickness of the fluid film $(m)$, and $u_{l}, u_{r}$ are the belt speed and the rate of change of fluid flow velocity on ended layer. We introduce the following dimensionless variables,

$$
\begin{gathered}
x^{*}=\frac{x}{\delta}, \\
v^{*}=\frac{v}{u_{l}}, \\
\beta=\frac{\left(\beta_{2}+\beta_{3}\right)}{\mu \delta^{2}} u_{l}, \\
m=\frac{\rho g}{\mu u_{l}} \delta^{2},
\end{gathered}
$$

where $l=\max \left\{u_{l}, u_{r}\right\}$. From Eqs.(4)-(7), we obtain the dimensionless from as (for simplicity we removed ${ }^{*}$ ),

$$
\frac{d^{2} v}{d x^{2}}+6 \beta\left(\frac{d v}{d x}\right)^{2} \frac{d^{2} v}{d x^{2}}-m=0, \text { for all } 0 \leq x \leq 1,
$$

Subject to the boundary conditions

$$
\begin{aligned}
& v(0)=v_{l}, \\
& v^{\prime}(1)=v_{r},
\end{aligned}
$$

where $v_{l}$ is the non-dimensional moving belts speed and $v_{r}$ is the rate of change of the fluid velocity on the ended layer.

\section{A Couple Numerical Techniques}

\subsection{A Centered in Space Finite Difference Method}

We will approximate the nonlinear ordinary differential equation model, which is a nonlinear boundary value problem with Dirichlet boundary conditions, Eqs.(8)-(10), by using a centered in spaced finite difference method. They can be written in a compact form,

$$
v^{\prime \prime}=\frac{m}{1+6 \beta\left(v^{\prime}\right)^{2}}, \text { for all } 0 \leq x \leq 1,
$$

We divide $[0,1]$ into $\mathrm{N}+1$ subintervals that endpoints are at $v_{i}=a+i h, \quad$ for all $i=0,1, \ldots, N+1$, where $h=1 /(N+1)$. By using the centered finite difference 
method [5], the finite difference equation can be written as follows,

$$
\frac{v_{i+1}-2 v_{i}+v_{i-1}}{h^{2}}=\frac{m}{1+6 \beta\left(\frac{v_{i+1}-v_{i-1}}{2 h}\right)^{2}},
$$

for all $i=0,1, \ldots, N$, It follows that

$$
\begin{gathered}
v_{i+1}+\frac{3}{2} \frac{\beta}{h^{2}}\left(v_{i+1}^{3}\right)-\frac{3}{2} \frac{\beta}{h^{2}}\left(v_{i+1}^{2} v_{i-1}\right)-\frac{3}{2} \frac{\beta}{h^{2}}\left(v_{i+1} v_{i-1}^{2}\right)-2 v_{i}-3 \frac{\beta}{h^{2}}\left(v_{i} v_{i+1}^{2}\right)+6 \frac{\beta}{h^{2}}\left(v_{i-1} v_{i} v_{i+1}\right) \\
-3 \frac{\beta}{h^{2}}\left(v_{i-1}^{2} v_{i}\right)+v_{i-1}+\frac{3}{2} \frac{\beta}{h^{2}}\left(v_{i-1}^{3}\right)=m h^{2}, \quad \text { (13) }
\end{gathered}
$$

for all $i=0,1, \ldots, N$, For $i=1$, plug the known value of the left boundary $v_{0}=u_{l}$ to Eq.(13) on the left hand side, we obtain

$\left(-2-3 \frac{\beta}{h^{2}}\right) v_{1}+\left(1-\frac{3}{2} \frac{\beta}{h^{2}}\right) v_{2}-\frac{3}{2} \frac{\beta}{h^{2}} v_{2}^{2}-\frac{3}{2} \frac{\beta}{h^{2}} v_{1} v_{2}^{2}+6 \frac{\beta}{h^{2}} v_{1} v_{2}+\frac{3}{2} \frac{\beta}{h^{2}} v_{2}^{3}=m h^{2}-v_{1}-\frac{3}{2} \frac{\beta}{h^{2}}$.

For $i=2,3, \ldots, N-1$, Eq.(13) becomes,

$$
\begin{array}{r}
v_{i-1}+\frac{3}{2} \frac{\beta}{h^{2}} v_{i-1}^{3}-\frac{3}{2} \frac{\beta}{h^{2}} v_{i-1}^{2} v_{i+1}-\frac{3}{2} \frac{\beta}{h^{2}} v_{i-1} v_{i+1}^{2}-2 v_{i}-\frac{3}{2} \frac{\beta}{h^{2}} v_{i} v_{i+1}^{2}-\frac{3}{2} \frac{\beta}{h^{2}} v_{i-1}^{2} v_{i}+6 \frac{\beta}{h^{2}} v_{i-1} v_{i} v_{i+1} \\
+v_{i+1}+\frac{3}{2} \frac{\beta}{h^{2}} v_{i+1}^{3}=m h^{2}
\end{array}
$$

To modify the finite difference method to a Neumann conditions, $\quad v^{\prime}(1)=\kappa$, if $i=N$, then Eq.(15) becomes,

$$
\begin{aligned}
& \left.2 h k+v_{N-1}+\frac{3}{2} \frac{\beta}{h^{2}}\left(2 h k+v_{N-1}\right)^{3}-\frac{3}{2} \frac{\beta}{h^{2}}\left(\left(2 h k+v_{N-1}\right)^{2} v_{N-1}\right)-\frac{3}{2} \frac{\beta}{h^{2}}\left(\left(2 h k+v_{N-1}\right)\right)_{N-1}^{2}\right)-2 v_{N} \\
& -3 \frac{\beta}{h^{2}}\left(v_{N}\left(2 h k+v_{N-1}\right)^{2}\right)+6 \frac{\beta}{h^{2}}\left(v_{N-1} v_{N}\left(2 h k+v_{N-1}\right)\right)-3 \frac{\beta}{h^{2}}+v_{N-1}+\frac{3}{2} \frac{\beta}{h^{2}}\left(v_{N-1}^{3}\right)=m h^{2} .
\end{aligned}
$$

\subsection{Quasi-Newton Iterative Method for a System of Nonlinear Finite Difference Equations}

Apply the Newton iterative to the system of simultaneous on nonlinear equation Eqs.(14-16) that can be written in a vector from as

$$
F(V)=\left(f_{1}\left(v_{1}, \ldots, v_{N}\right), f_{2}\left(v_{2}, \ldots, v_{N}\right), \ldots, f_{N}\left(v_{1}, \ldots, v_{N}\right)\right)^{t},
$$

where

$$
\begin{gathered}
f_{1}\left(v_{1}, \ldots, v_{N}\right):=\left(-2-3 \frac{\beta}{h^{2}}\right) v_{1}+\left(1-\frac{3}{2} \frac{\beta}{h^{2}}\right) v_{2}-\frac{3}{2} \frac{\beta}{h^{2}} v_{2}^{2}-\frac{3}{2} \frac{\beta}{h^{2}} v_{1} v_{2}^{2}+6 \frac{\beta}{h^{2}} v_{1} v_{2}+\frac{3}{2} \frac{\beta}{h^{2}} v_{2}^{3} \\
-\left(m h^{2}-v_{1}-\frac{3}{2} \frac{\beta}{h^{2}}\right), \\
f_{i}\left(v_{1}, \ldots, v_{N}\right):=v_{i-1}+\frac{3}{2} \frac{\beta}{h^{2}} v_{i-1}^{3}-\frac{3}{2} \frac{\beta}{h^{2}} v_{i-1}^{2} v_{i+1}-\frac{3}{2} \frac{\beta}{h^{2}} v_{i-1} v_{i+1}^{2}-2 v_{i}-\frac{3}{2} \frac{\beta}{h^{2}} v_{i} v_{i+1}^{2}-\frac{3}{2} \frac{\beta}{h^{2}} v_{i-1}^{2} v_{i} \\
+6 \frac{\beta}{h^{2}} v_{i-1} v_{i} v_{i+1}+v_{i+1}+\frac{3}{2} \frac{\beta}{h^{2}} v_{i+1}^{3}-m h^{2},
\end{gathered}
$$

for all $i=2,3, \ldots, N-1$,

$f_{N}\left(v_{1}, \ldots, v_{N}\right):=2 h k+v_{N-1}+\frac{3}{2} \frac{\beta}{h^{2}}\left(2 h k+v_{N-1}\right)^{3}-\frac{3}{2} \frac{\beta}{h^{2}}\left(\left(2 h k+v_{N-1}\right)^{2} v_{N-1}\right)$

$\left.-\frac{3}{2} \frac{\beta}{h^{2}}\left(2 h k+v_{N-1}\right) v_{N-1}^{2}\right)-2 v_{N}-3 \frac{\beta}{h^{2}}\left(v_{N}\left(2 h k+v_{N-1}\right)^{2}\right)+6 \frac{\beta}{h^{2}}\left(v_{N-1} v_{N}\left(2 h k+v_{N-1}\right)\right)$

$$
-3 \frac{\beta}{h^{2}}+v_{N-1}+\frac{3}{2} \frac{\beta}{h^{2}}\left(v_{N-1}^{3}\right)-m h^{2} .
$$

The Jacobian matrix $J(V)$ for the system of Eqs.(17-19) is given by

$$
J(V)=\left[\begin{array}{cccc}
\partial f_{1} / \partial v_{1} & \partial f_{2} / \partial v_{1} & \cdots & \partial f_{1} / \partial v_{N} \\
\partial f_{2} / \partial v_{1} & \partial f_{2} / \partial v_{2} & \cdots & \partial f_{2} / \partial v_{N} \\
\vdots & \vdots & \\
\partial f_{N} / \partial v_{1} & \partial f_{N} / \partial v_{2} & \cdots & \partial f_{N} / \partial v_{N}
\end{array}\right] .
$$

Choosing that $V^{0}=\left(v_{1}^{(0)}, \ldots, v_{N}^{(0)}\right)^{t}$, we can obtain $F\left(V^{(0)}\right)$ and $J\left(V^{(0)}\right)$. Then we will solve the obtained linear system,

$$
J\left(V^{(0)}\right) U^{(0)}=-F\left(V^{(0)}\right),
$$

we get $U^{(0)}$. Then

$$
V^{(1)}=V^{(0)}+U^{(0)} .
$$

To continuing in this process for $k=2,3, \ldots$ we have

$$
V^{(2)}=V^{(1)}+A_{1}^{-1} F\left(V^{(1)}\right) .
$$

So $V^{(2)}$ is also determined, the method is repeated to determine $V^{(3)}$, using $A_{(1)}$ in place of $A_{(0)}=J\left(V^{0}\right)$, and with $V^{(2)}$ and $V^{(1)}$ in place of $V^{(1)}$ and $V^{(0)}$. In general, once $V^{(i)}$ has been determined, $V^{(i+1)}$ is computed by

$$
A_{i}=A_{i-1}+\frac{y_{i}-A_{i-1} s_{i}}{\left\|s_{i}\right\|_{2}^{2}} s_{i}^{t}
$$

and

$$
V^{(i+1)}=V^{(i)}+A_{i}^{-1} F\left(V^{(i)}\right),
$$

where the notation $y_{i}=F\left(V^{(i)}\right)-F\left(V^{(i-1)}\right)$ and $s_{i}=V^{(i)}-V^{(i-1)}$ is introduced to simplify the equations.

$$
A_{i} S_{i+1}=-F\left(V^{(i)}\right) .
$$

\section{Numerical Simulation}

Assuming that there is a moving belt in a third grade fluid basis . The basin is filled by the engine oil SAE $15 \mathrm{~W}-40$ with the viscosity, $31.350 \mathrm{~mm}^{2} / \mathrm{s}$ and the density, $0.8477 \mathrm{~g} / \mathrm{cm}^{3}$ at $70^{\circ} \mathrm{C}$. The material constant of the engine oil is $1.5675 \times 10^{-5}$. The moving belt has speed $1 \mathrm{~m} / \mathrm{s}$ and the uniform thickness of fluid film is 
$0.001 \mathrm{~m}$.We will consider physical parameters in 4 cases as show in Table 1 .By using the finite difference equations Eqs.(14-16) with the Quasi-Newton iterative techniques Eqs.(17-19), we can obtain the thin fluid film flow velocity in each layers .If the speed of moving belt is uniform and the rates of change of fluid film flow velocity over the ended layer are nonnegative due to external force, the approximated flow velocity in each layers are show in Table 1 and Figure 1. However, the cases of rates of change of fluid film flow velocity over the ended layer are negative due to gravity force and some external force are also investigated as show in Table 2 and Figure 2.

Table 1. Comparison of nondimensional thin film flow velocity in each nonnegative rates of change with $m=0.265$ and $\beta=0.5$.

\begin{tabular}{|c|c|c|c|c|}
\hline$x \backslash v_{r}$ & 0.0000 & 0.00025 & 0.0005 & 0.0010 \\
\hline 0.0 & 1.0000 & 1.0000 & 1.0000 & 1.0000 \\
\hline 0.1 & 0.9762 & 0.9762 & 0.9762 & 0.9763 \\
\hline 0.2 & 0.9546 & 0.9547 & 0.9547 & 0.9548 \\
\hline 0.3 & 0.9355 & 0.9355 & 0.9356 & 0.9357 \\
\hline 0.4 & 0.9187 & 0.9188 & 0.9189 & 0.9191 \\
\hline 0.5 & 0.9044 & 0.9045 & 0.9047 & 0.9049 \\
\hline 0.6 & 0.8927 & 0.8928 & 0.8929 & 0.8932 \\
\hline 0.7 & 0.8835 & 0.8836 & 0.8839 & 0.8841 \\
\hline 0.8 & 0.8769 & 0.8771 & 0.8772 & 0.8776 \\
\hline 0.9 & 0.8729 & 0.8731 & 0.8733 & 0.8737 \\
\hline 1.0 & 0.8716 & 0.8718 & 0.8720 & 0.8725 \\
\hline
\end{tabular}

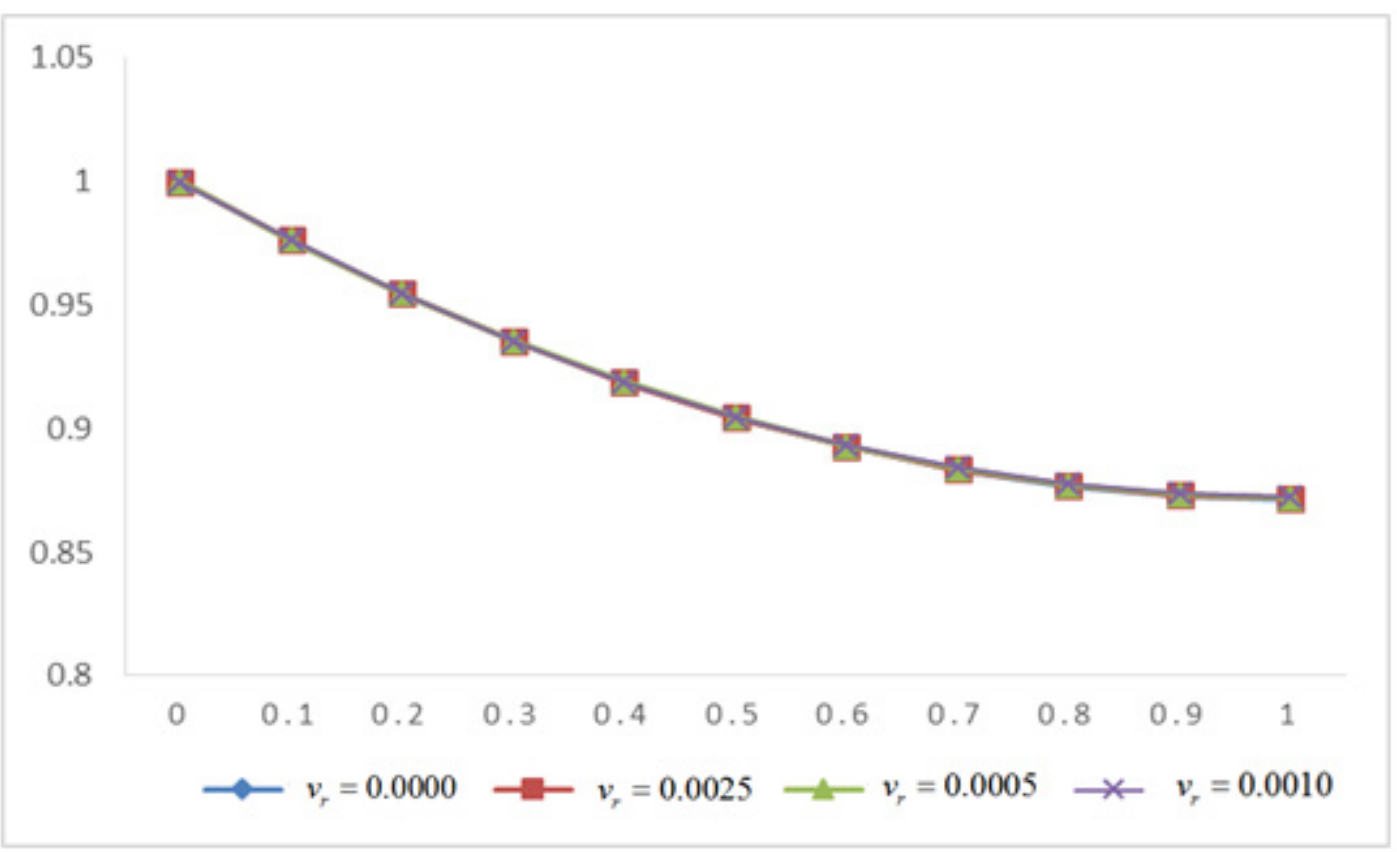

Figure 1. Comparison of nondimensional thin film flow velocity in each nonnegative rates of change with $m=0.265$ and $\beta=0.5$. 
A Mathematical Model of Lubricant Film Flow Velocity on a Belt Type Oil Skimmer in a Part of Wastewater Treatment Process Using a Finite Difference Method with Quasi-Newton Iterative Technique

Table 2. Comparison of nondimensional thin film flow velocity in each negative rates of change with $m=0.265$ and $\beta=0.5$.

\begin{tabular}{|c|c|c|c|}
\hline$x \backslash v_{r}$ & -0.00100 & -0.00050 & -0.00025 \\
\hline 0.0 & 1.0000 & 1.0000 & 0.9762 \\
\hline 0.1 & 0.9761 & 0.9761 & 0.9546 \\
\hline 0.2 & 0.9545 & 0.9546 & 0.9354 \\
\hline 0.3 & 0.9352 & 0.9353 & 0.9186 \\
\hline 0.4 & 0.9184 & 0.9185 & 0.9043 \\
\hline 0.5 & 0.9040 & 0.9042 & 0.8925 \\
\hline 0.6 & 0.8921 & 0.8924 & 0.8833 \\
\hline 0.7 & 0.8828 & 0.8831 & 0.8767 \\
\hline 0.8 & 0.8761 & 0.8765 & 0.8727 \\
\hline 0.9 & 0.8721 & 0.8725 & 0.8713 \\
\hline
\end{tabular}

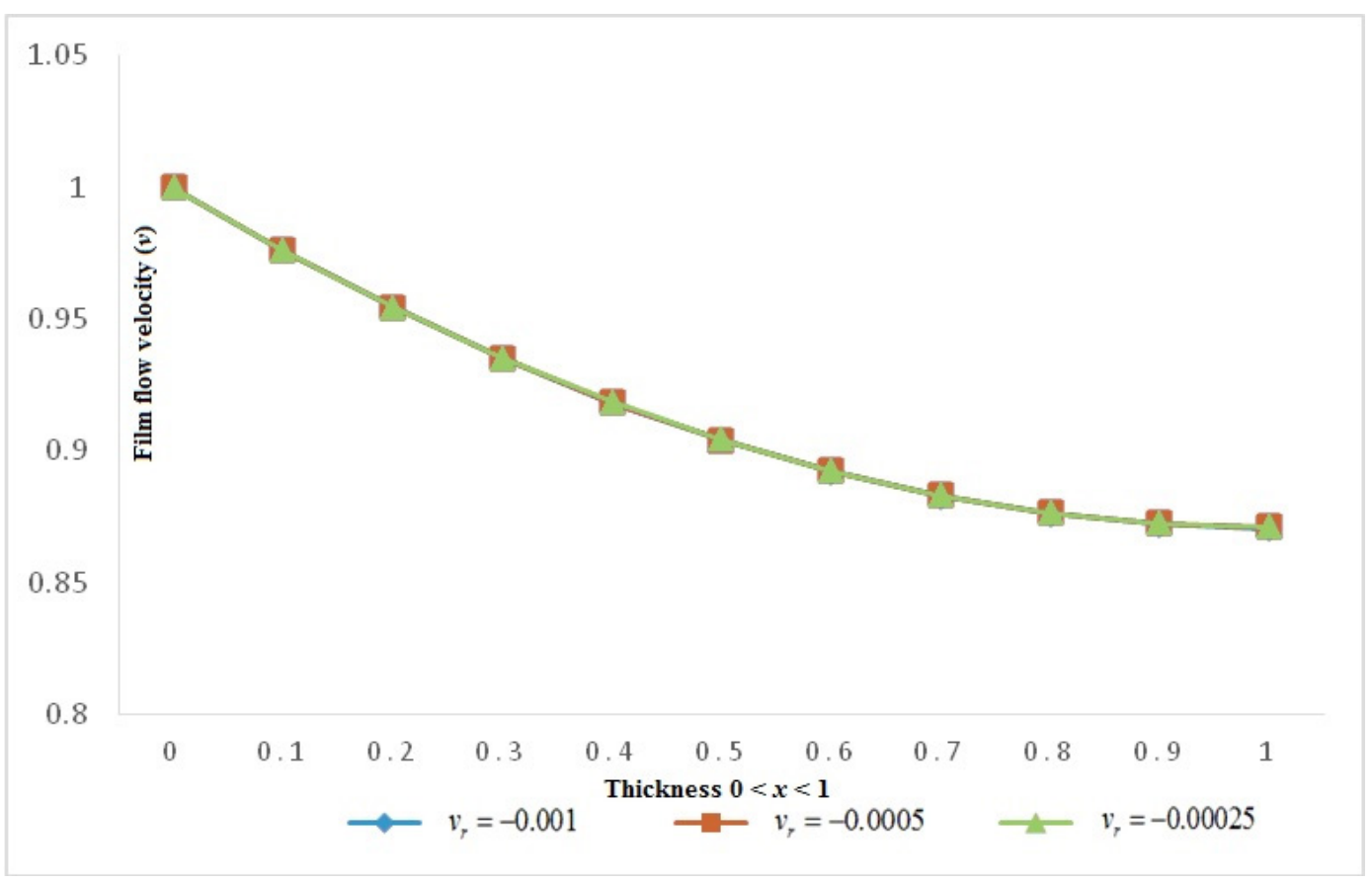

Figure 2. Comparison of nondimensional thin film flow velocity in each negative rates of change with $m=0.265$ and $\beta=0.5$. 


\section{Conclusions}

A centered in space finite difference method with the Quasi-Newton iterative technique is employed to simulate lubricant film flow velocity on a moving belt oil skimmer models in several physical parameters. The results are shown that the thin fluid film flow velocity of motor oil can be change when the external force becomes positive or negative. If the external force is increasing, the fluid film flow velocities at all layers are also increased as well.

\section{Acknowledgments}

This research is supported by the Centre of Excellence in Mathematics, the Commission on Higher Education, Thailand.

\section{REFERENCES}

[1] Siddiqui, A. M., Mahmood, R. and Ghori, Q. K. Thin film flow of a third grade fluid on a moving belt by homotopy perturbation method, International Journal of Nonlinear Sciences and Numerical Simulation, Vol.7(1), 2006, 7-14.

[2] Siddiqui, A.M., Mahmood, R. and Ghori, Q. K., Homotopy perturbation method for thin film flow of a third grade fluid down an inclined plane", Chaos, Solitons \& Fractals, Vol.35(1), 2008, 140-147.

[3] Khan, R. A., (2010) "Generalized approximation method and a thin film flow of a third grade fluid on a moving belt", Computational Mathematics and Modeling, Vol. 21(1), 2010, 41-50.

[4] Siddiqui, A. M., Farooq, A.A., Haroon, T. and Babcock, B.S., A comparison of variational iteration and Adomian decomposition methods in solving nonlinear thin film flow problems, Applied Mathematical Sciences, Vol.6(97), 2012, 4911-4919.

[5] Mabood, F. and Pochai, N., "Comparison of Optimal Homotopy Asymptotic and Adomian Decomposition Methods for a Thin Film Flow of a Third Grade Fluid on a Moving Belt", Advances in Mathematical Physics, 2015, Article ID 642835, 4 pages.

[6] Burden, R.L. and Faires, J.D., Numerical Analysis, Thomson Brooks/Cole, Singapore, 2005.

[7] Fazle M, Pochai N and Shateyi S, Stagnation Point Flow of Nanofluid over a Moving Plate with Convective Boundary Condition and Magnetohydrodynamics, Journal of Engineering, Volume 2016, Article ID 5874864, 11 pages, 2016.

[8] Mabood F, Lorenzini G, Pochai N, Muhammad S, Effects of prescribed heat flux and transpiration on MHD axisymmetric flow impinging on stretching cylinder, Continuum Mechanics and Thermodynamics, Volume 28, Issue 6, 1925-1932, 2016.

[9] Hayat T, Mambili-Mamboundou H and Mahomed F. M.,
Unsteady Solutions in a Third-Grade Fluid Filling the Porous Space, Mathematical Problems in Engineering, Volume 2008, Article ID 139560, 13 pages, 2008.

[10] Zeb M., Islam S., Siddiqui A. M. and Haroon T., Analysis of Third-Grade Fluid in Helical Screw Rheometer, Journal of Applied Mathematics, Volume 2013, Article ID 620238, 11 pages, 2013. 